\title{
Mass Measurement Using the Fixed Point of a Spring-Mass System with a Dynamic Vibration Absorber*
}

\author{
Satoru YAMAMOTO**, Yuji ISHINO**, Masaya TAKASAKI** \\ and Takeshi MIZUNO** \\ **Department of Mechanical Engineering, Saitama University \\ Shimo-Okubo 255, Sakura-ku, Saitama 338-8570, Japan \\ E-mail: s10mh222@mail.saitama-u.ac.jp
}

\begin{abstract}
A vibration-type measurement system characterized by the use of an undamped dynamic vibration absorber has been developed. However, inevitable damping in the absorber may cause measurement error. A new method of measuring mass is proposed to overcome this problem. The measurement system utilizes the fixed point of a mass-spring system with a dynamic vibration absorber so that the mass is estimated regardless of damping in the absorber. A phase-looked loop (PLL) is used to achieve tuning. The principle of measurement is described on the basis of a mathematical model. A measuring apparatus was designed and fabricated, and several of its basic characteristics were studied experimentally. Damping of the primary system was found to affect fixed point formation. By reducing the damping of the primary system by a voice coil motor, the measurement conditions were achieved. The efficacy of the apparatus was studied both analytically and experimentally. The measurement conditions were realized automatically by the PLL. Mass measurement was performed while the PLL was operated; the average measurement error was within $0.21[\%]$.
\end{abstract}

Key words: Mass Measurement, Dynamic Vibration Absorber, Vibration Control Phase-Looked Loop, Space Engineering

\section{Introduction}

The completion of the International Space Station will increase the opportunities for experiments in space. The necessity for mass measurement under weightless conditions will increase accordingly. Various methods of measuring mass under weightless conditions have been proposed $[1,2]$. The authors have proposed the use of a dynamic vibration absorber for both mass measurement and vibration control [3-5]. Mass measurement systems with a vibration absorber are classified into centrifugal and vibration types, according to the motion of the object to be measured [6]. In the centrifugal type, the object to be measured is fixed to a rotating table (rotor) at a distance from the rotational axis [3-5]. Because the object unbalances the rotor, centrifugal forces cause the supporting structure to vibrate during rotation. A dynamic vibration absorber attached to the structure is tuned to stop the vibration. When the structure does not vibrate, the absorber vibrates in such a way that the product of the absorber mass and the amplitude of its vibration equals the amount of unbalance, that is, the product of the mass to be measured and its distance from the rotational axis. Therefore, the mass of the object can be determined from the motion of the absorber mass. In the vibration type, an object is attached to the absorber mass of a dynamic 
vibration absorber instead of a rotating table [7, 8]. One of the advantages of the vibration type over the centrifugal type is that it can estimate mass regardless of the place at which a measurement object is fixed.

We have developed a vibration-type measurement system characterized by the use of an undamped dynamic vibration absorber [9, 10]. However, inevitable damping in the absorber may cause measurement error. A new method of measuring mass is proposed to overcome this problem. The frequency response curves of a mass-spring system with a dynamic vibration absorber pass through a fixed point that is independent of damping in the absorber. The proposed method utilizes this fixed point so that mass is estimated regardless of the damping. The measurement principle is described on the basis of a mathematical model. A measurement apparatus is designed and fabricated, and several of its basic characteristics are studied experimentally. The efficacy of the apparatus is studied both analytically and experimentally. Mass measurements with the fabricated apparatus are described.

\section{Principle of measurement}

A basic model of the proposed mass measurement system with a damped dynamic vibration absorber is shown in Fig.1. The primary system consists of a primary mass $m_{p}$ and a spring $k_{p}$. A dynamic vibration absorber consisting of an absorber of mass $m_{a}$, a spring $k_{a}$, and a damper $c_{a}$ is attached to the primary mass. When the force acting on the primary mass is represented by $f(t)$, the equations of motion are derived as

$$
\begin{aligned}
& m_{p} \ddot{x}_{p}=-k_{p} x_{p}-k_{a}\left(x_{p}-x_{a}\right)-c_{a}\left(\dot{x}_{p}-\dot{x}_{a}\right)+f(t), \\
& m_{a} \ddot{x}_{a}=-k_{a}\left(x_{a}-x_{p}\right)-c_{a}\left(\dot{x}_{a}-\dot{x}_{p}\right) .
\end{aligned}
$$

From Eqs. (1) and (2), we get

$$
\begin{aligned}
& G_{p}(s) \equiv \frac{X_{p}(s)}{F(s)}=\frac{t_{a}(s)}{t_{p}(s) t_{a}(s)+\left(c_{a} s+k_{a}\right) m_{a} s^{2}}, \\
& G_{a}(s) \equiv \frac{X_{a}(s)}{F(s)}=\frac{c_{a} s+k_{a}}{t_{p}(s) t_{a}(s)+\left(c_{a} s+k_{a}\right) m_{a} s^{2}},
\end{aligned}
$$

where

$$
\begin{aligned}
& t_{p}(s)=m_{p} s^{2}+k_{p}, \\
& t_{a}(s)=m_{a} s^{2}+c_{a} s+k_{a} .
\end{aligned}
$$

To obtain the stationary vibration displacements when the force $f(t)$ is harmonic, we represent the variables as

$$
f(t)=F e^{j \omega t}, \quad x_{p}(t)=\bar{X}_{p} e^{j \omega t}, x_{a}(t)=\bar{X}_{a} e^{j \omega t} .
$$

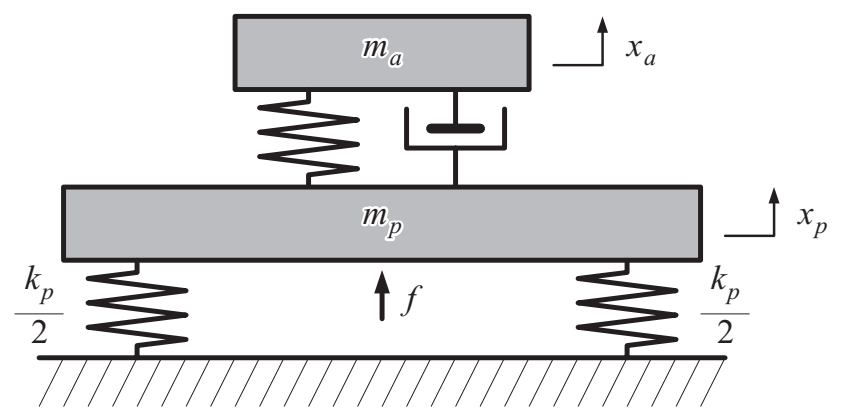

Fig.1 Basic model of mass measurement system with a damped dynamic vibration absorber 
The complex constants (phasors) $\bar{X}_{p}$ and $\bar{X}_{a}$ are given by

$$
\begin{aligned}
& \bar{X}_{p}=G_{p}(j \omega) F, \\
& \bar{X}_{a}=G_{a}(j \omega) F .
\end{aligned}
$$

The behavior of the primary mass has been studied extensively. According to the well-known fixed points theorem, all the curves in the frequency response diagram pass through two points independent of the damping [11]. This theorem has been utilized to optimize the damper. In contrast, we focus on the behavior of the absorber mass in this research.

Figure 2 shows a plot of the vibration amplitude of the absorber mass as a function of the angular frequency for various values of the damping $c_{a}$. Figure 3 shows an expanded plot near the fixed point. The values of $m_{p}, k_{p}, m_{a}$, and $k_{a}$ are selected to correspond to those of the fabricated apparatus, $m_{p}=0.599[\mathrm{~kg}], k_{p}=8.74 \times 10^{3}[\mathrm{~N} / \mathrm{m}], m_{a}=0.585[\mathrm{~kg}]$, and $k_{a}$ $=8.85 \times 10^{3}[\mathrm{~N} / \mathrm{m}]$ (see Table 1 ). In this figure, $0[\mathrm{~dB}]$ corresponds to $1[\mathrm{~m}]$ when $1[\mathrm{~N}]$ is added. This figure shows that the fixed point exists for all the curves regardless of damping in the absorber. It will be shown analytically that all the curves pass through a point independent of the damping.

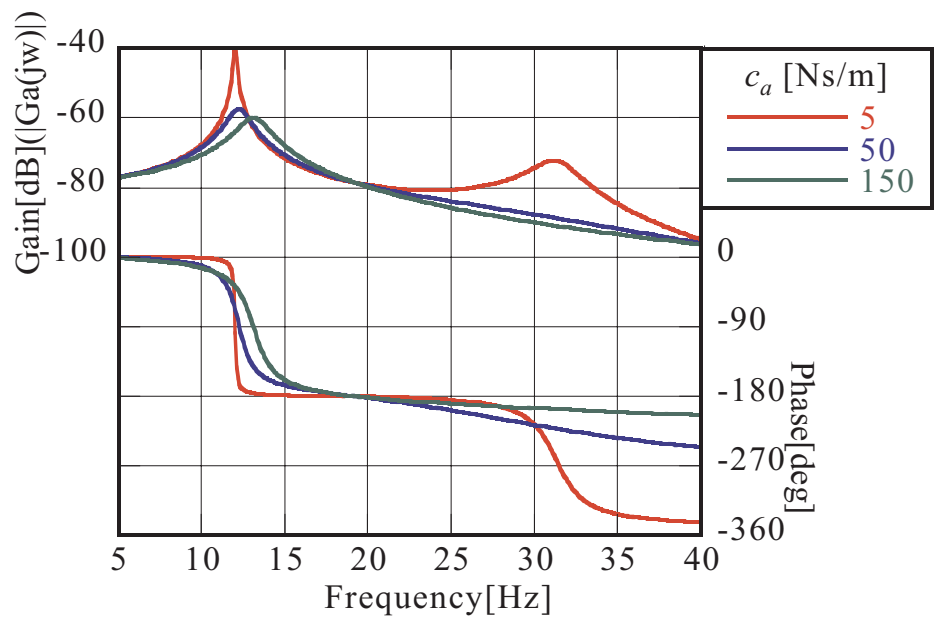

Fig.2 Calculated frequency responses

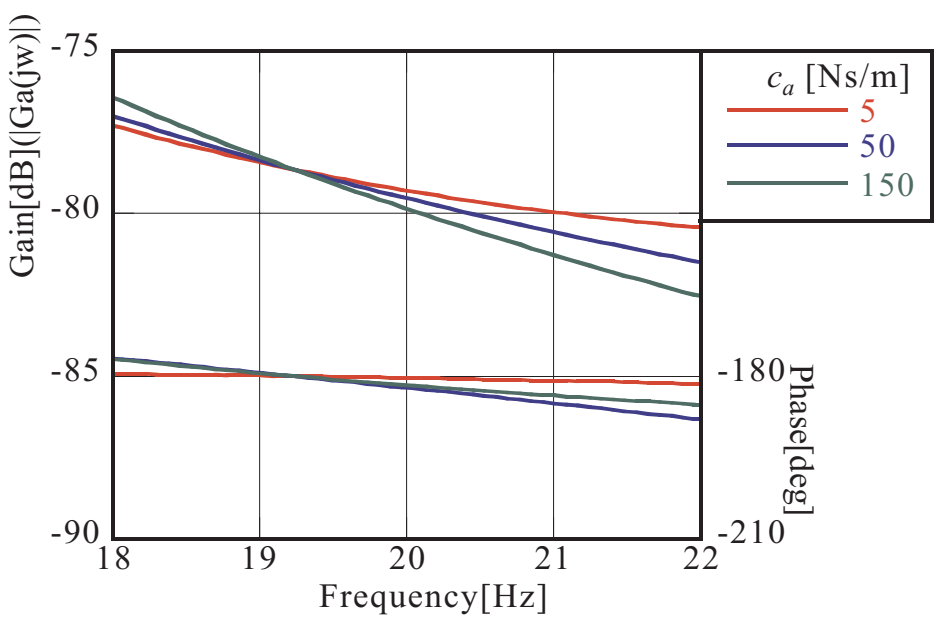

Fig.3 Expanded plot near the fixed point 
We define the resonant frequency of the primary system as

$$
\omega_{p} \equiv \sqrt{\frac{k_{p}}{m_{p}}} .
$$

The frequency of the harmonic force is assumed to be equal to this frequency, that is,

$$
\omega=\omega_{p} \text {. }
$$

From Eq. (4), we get

$$
\bar{X}_{a}=-\frac{m_{p}}{m_{a}} \cdot \frac{F}{k_{p}} .
$$

Equation (12) indicates that:

- The vibration amplitude of the absorber mass is independent of the damping $c_{a}$.

- The phase difference between the force and displacement of the absorber mass is just 180 degrees.

These relationships are critical for constructing the proposed mass measurement system.

A measurement object $\Delta m_{a}$ is attached to the absorber mass, from Eq. (12),

$$
\Delta m_{a}=\frac{m_{p} F}{\left|\bar{X}_{a}\right| k_{p}}-m_{a},
$$

when Eq. (11) is satisfied. Equation (13) indicates that the mass of an object attached to the absorber mass can be determined by measuring the vibration amplitude of the absorber mass.

Mass can also be determined by attaching an object to the primary mass instead of the absorber mass because the following equation is derived from Eq. (12).

$$
\Delta m_{p}=m_{a}\left|\bar{X}_{a}\right| \frac{k_{p}}{F}-m_{p},
$$

where $\Delta m_{p}$ represents a measurement object attached to the primary mass $m_{p}$. In this case, mass can also be determined by measuring the tuned frequency because the following equation can be derived from Eqs. (10) and (11).

$$
\Delta m_{p}=\frac{k_{p}}{\omega^{2}}-m_{p}
$$

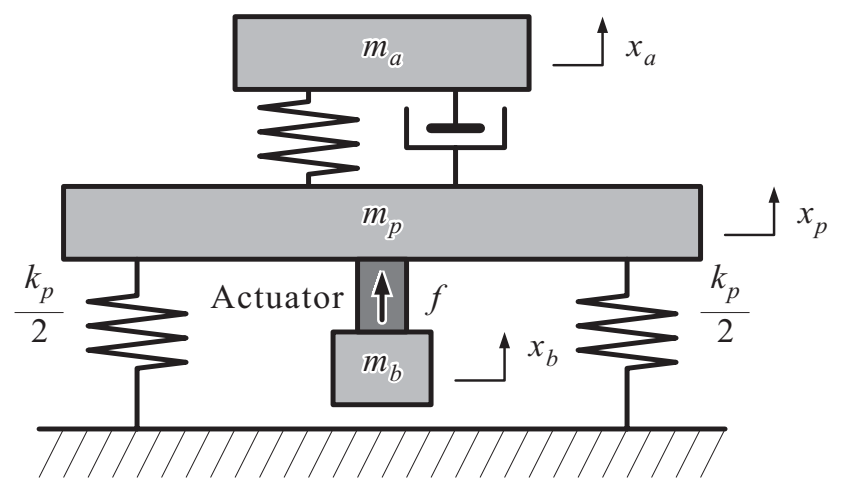

Figure 4. Another model of mass measurement system with an inertial mass 
Figure 4 shows another configuration of the proposed mass measurement system. The harmonic force acting on the primary mass is produced by a vibrator, consisting of an actuator and an inertial mass $m_{b}$. The equation of motion of the inertial mass is given by

$$
m_{b} \ddot{x}_{b}=-f(t) \text {. }
$$

To obtain the steady-state solution, we assume that

$$
x_{b}(t)=\bar{X}_{b} e^{j \omega t} \text {. }
$$

The complex constant $X_{b}$ is given by

$$
\bar{X}_{b}=\frac{F}{m_{b} \omega^{2}} .
$$

The measured object attached to mass $m_{b}$ is $\Delta m_{b}$. From Eqs. (10), (11), and (14),we get

$$
\Delta m_{b}=m_{a}\left|\frac{\bar{X}_{a}}{\bar{X}_{b}}\right|-m_{b},
$$

or

$$
\Delta m_{a}=m_{b}\left|\frac{\bar{X}_{a}}{\bar{X}_{b}}\right|-m_{a} .
$$

Equations (19) and (20) indicate that a measurement object can be attached to either the absorber mass or the inertial mass. In both cases, the mass can be determined by measuring the ratio of the vibration amplitudes of the absorber mass and the inertial mass.

Equations (19) and (20) also indicate that the phase difference between the absorber mass and the inertial mass is just 180 degrees when Eq. (11) is satisfied.

\section{Measurement system}

The apparatus shown in Fig.1 was fabricated. To estimate mass according to Eq. (13), the condition given by Eq. (11) must be satisfied. To achieve this condition, a phase-locked loop (PLL) was applied in this research.

A typical PLL is a circuit that synchronizes an output signal generated by an oscillator with a reference or input signal in frequency as well as in phase [12]. It consists of three functional blocks:

- Voltage-controlled oscillator (VCO)

- Phase detector (PD)

- Loop filter (LF)

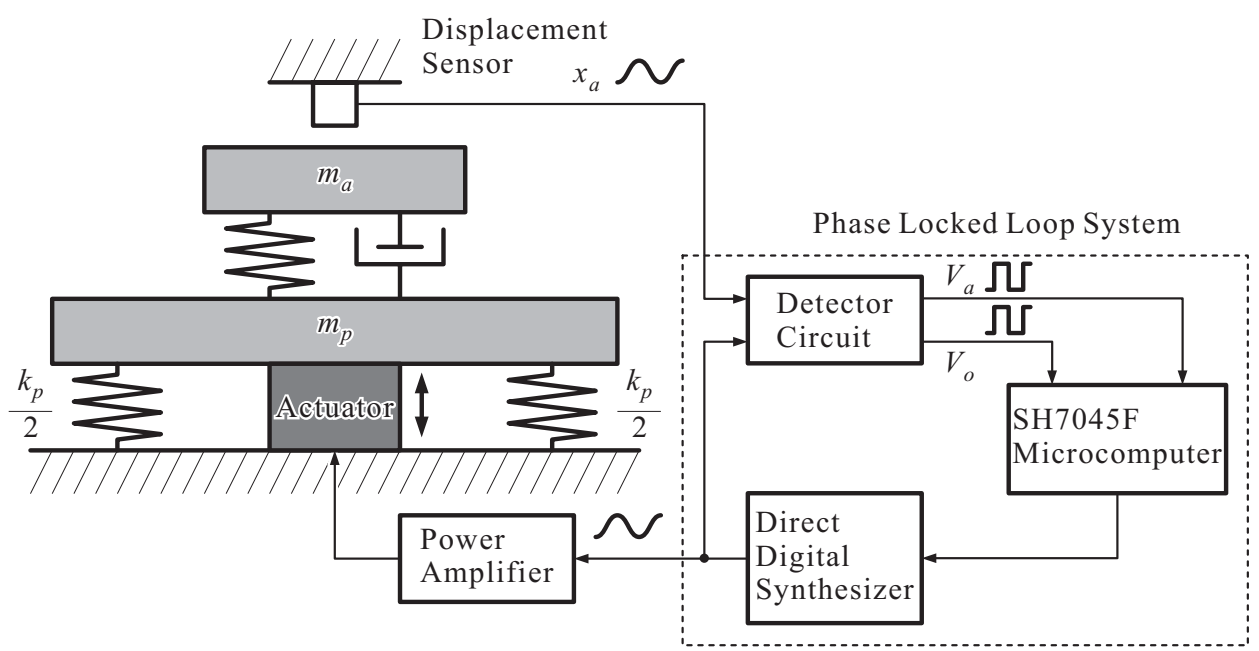

Figure 5. Control and measurement system with direct excitation 
The PLL has broad industrial applications in telecommunications, electrical measurement, and frequency synthesis. It is also applied to the precise control of motor speed [13] and self-sensing control of magnetic suspension [14]. However, mass measurement using a PLL has not been reported.

As mentioned above, the phase difference between the force and the displacement of the absorber mass is just 180 degrees when Eq. (11) is satisfied. The frequency of the force is tuned to realize this phase condition according to the output of the PD, which is a key component of the PLL.

Figure 5 shows the configuration of the proposed mass measurement system. The harmonic force is produced by an actuator with force output, which is mounted directly on the base. In this configuration, it is assumed that no phase difference exists between the force and the output of the VCO, another key component of the PLL.

\section{Experiment}

\subsection{Experiment system}

The apparatus was fabricated to verify the measurement principle. Its parameters are shown in Table 1. Figures 6 and 7 show a photo of the fabricated apparatus and a schematic drawing, respectively. Both the primary mass $m_{p}$ and the absorber mass $m_{a}$ vibrate horizontally. The primary mass $m_{p}$ is T-shaped and suspended by a pair of leaf springs attached to the base. The absorber mass $m_{a}$ is also suspended by a pair of leaf springs attached to the primary mass. The absorber mass is driven by a voice coil motor (VCM), which is used to adjust the stiffness $k_{a}$ and add damping $c_{a}$. Another VCM fixed to the base produces a force $f$ acting on the primary mass. The primary mass displacement $x_{p}$ and the absorber mass displacement $x_{a}$ are measured by laser-type displacement sensors (KEYENCE LCP-2440).

Table 1 System parameters

\begin{tabular}{cc|cc}
\hline \multicolumn{2}{c|}{ Primary system } & \multicolumn{2}{c}{ Absorber } \\
\hline \hline$m_{p}$ & $0.599[\mathrm{~kg}]$ & $m_{a}$ & $0.585[\mathrm{~kg}]$ \\
$k_{p}$ & $8.74 \times 10^{3}[\mathrm{~N} / \mathrm{m}]$ & $k_{a}$ & $8.85 \times 10^{3}[\mathrm{~N} / \mathrm{m}]$ \\
& & $c_{a}$ & $0.764[\mathrm{Ns} / \mathrm{m}]$ \\
& & $\zeta_{\mathrm{a}}$ & 0.00311 \\
\hline
\end{tabular}

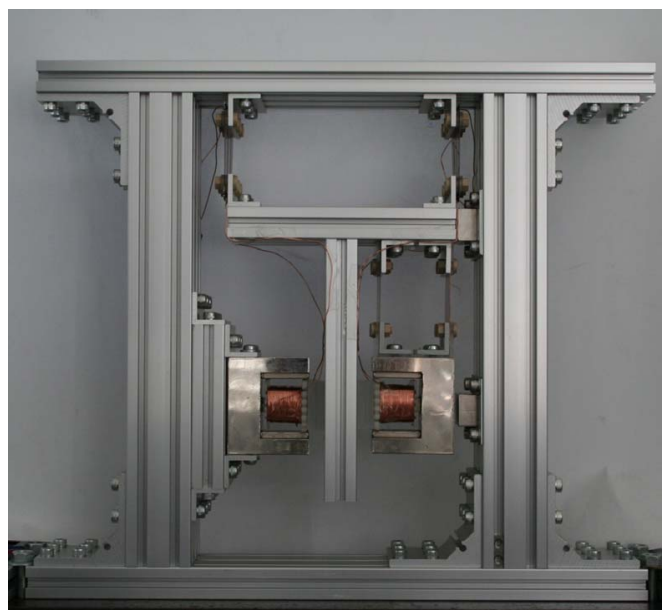

Fig.6 Measuring apparatus 


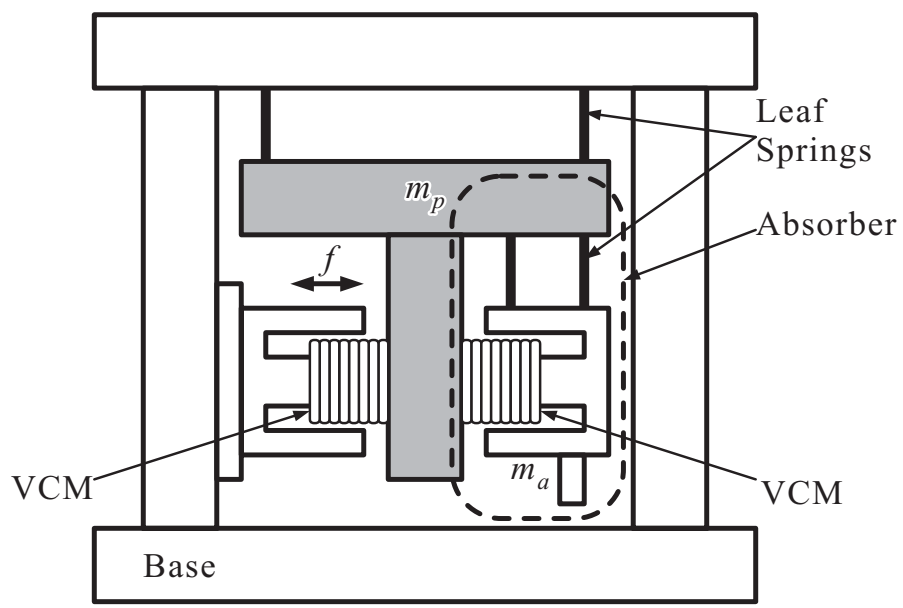

Fig.7 Schematic drawing of the measuring apparatus

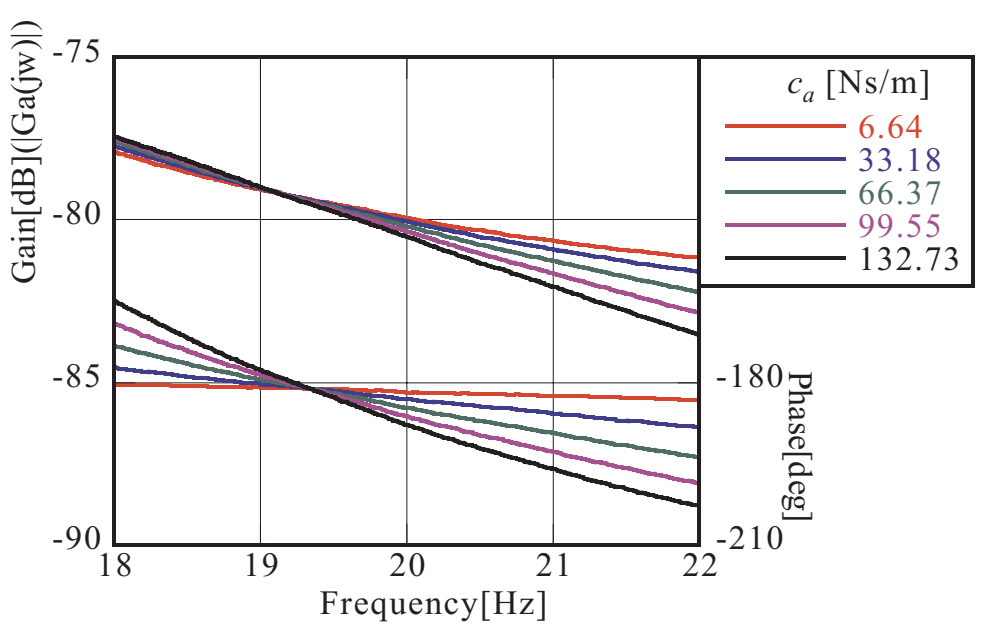

Fig.8 Measured frequency response

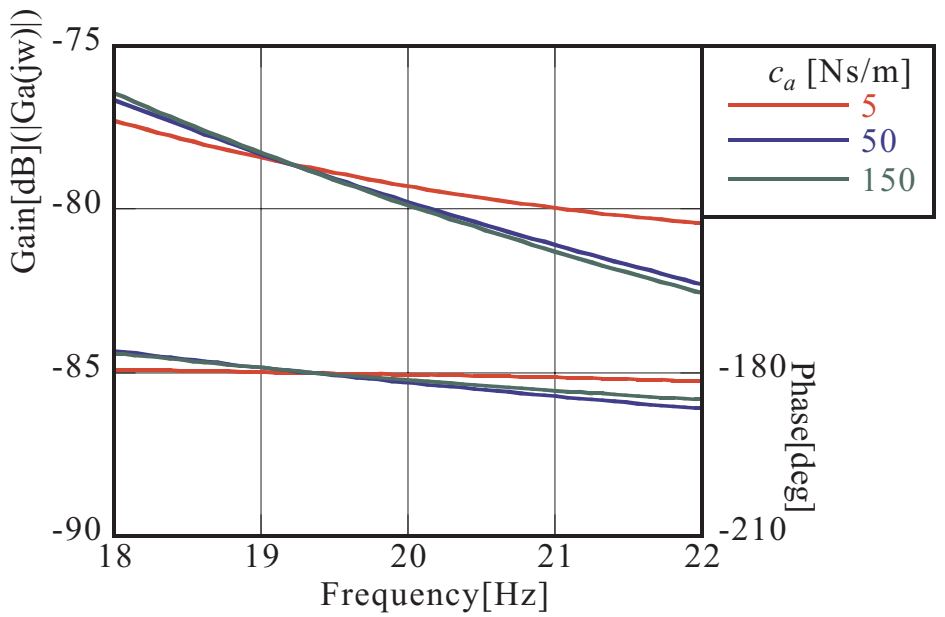

Fig.9 Calculated frequency responses with damping in the primary system

\subsection{Frequency response}

Figure 8 shows frequency response curves of the absorber mass displacement as a function of the angular frequency for various values of $c_{a}$. The fixed point of the phase 
curves is at $19.4[\mathrm{~Hz}]$, whereas that of the gain curves is at $19.1[\mathrm{~Hz}]$. This difference in the fixed points may be due to the primary system damping $c_{p}$, which is analyzed in the next section.

\subsection{Effect of damping in the primary system}

When damping exists in the primary system, the equations of motion become

$$
\begin{aligned}
& m_{p} \ddot{x}_{p}=-k_{p} x_{p}-c_{p} \dot{x}_{p}-k_{a}\left(x_{p}-x_{a}\right)-c_{a}\left(\dot{x}_{p}-\dot{x}_{a}\right)+f(t), \\
& m_{a} \ddot{x}_{a}=-k_{a}\left(x_{a}-x_{p}\right)-c_{a}\left(\dot{x}_{a}-\dot{x}_{p}\right) .
\end{aligned}
$$

From Eqs. (21) and (22), we get

$$
\begin{aligned}
\frac{X_{p}(s)}{F(s)} & =\frac{t_{a}(s)}{\widetilde{t}_{p}(s) t_{a}(s)+\left(c_{a} s+k_{a}\right) m_{a} s^{2}}, \\
\frac{X_{a}(s)}{F(s)} & =\frac{c_{a} s+k_{a}}{\widetilde{t}_{p}(s) t_{a}(s)+\left(c_{a} s+k_{a}\right) m_{a} s^{2}},
\end{aligned}
$$

where

$$
\begin{gathered}
\tilde{t}_{p}(s)=m_{p} s^{2}+c_{p} s+k_{p}, \\
t_{a}(s)=m_{a} s^{2}+c_{a} s+k_{a}
\end{gathered}
$$

When Eq. (11) is satisfied, it is derived from Eq. (24) that

$$
G_{a}\left(j \omega_{p}\right)=\frac{X_{a}\left(j \omega_{p}\right)}{F\left(j \omega_{p}\right)}=\frac{j c_{a} \omega_{p}+k_{a}}{\widetilde{t}_{p}\left(j \omega_{p}\right) t_{a}\left(j \omega_{p}\right)-\left(j c_{a} \omega_{p}+k_{a}\right) m_{a} \omega_{p}{ }^{2}},
$$

where

$$
\tilde{t}_{p}\left(j \omega_{p}\right)=j c_{p} \omega_{p},
$$

which is generally nonzero.

Figure 9 shows a plot of the vibration amplitude of the absorber mass as a function of the angular frequency for various values of the damping $c_{a}$ when the primary system damping $c_{p}$ is $0.5[\mathrm{Ns} / \mathrm{m}]$. The fixed point of the phase curves is at $19.4[\mathrm{~Hz}]$, whereas that of the gain curves is lower, i.e., 19.1 [Hz]. These results agree well with the experimental results shown in Fig.8. In addition, it can be shown that the fixed points vanish when the primary system damping $c_{p}$ is rather large.

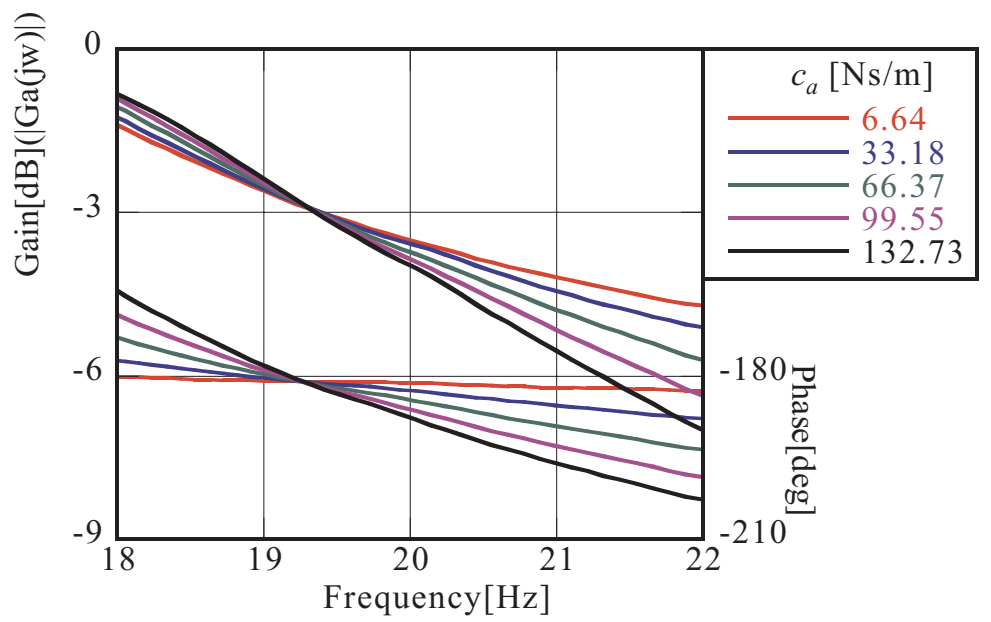

Fig.10 Measured frequency response with decreasing damping $c_{p}$ 


\subsection{Reduction in damping}

In the measuring apparatus, the primary system damping $c_{p}$ was reduced by the VCM for producing a harmonic force by feeding back the velocity of the primary mass. Figure 10 shows the frequency response curves of the absorber mass as a function of the angular frequency for various values of $c_{a}$. The fixed points of the gain and the phase curves appear at almost the same frequency. Therefore, the conditions for measurement have been realized, and measurement can be conducted according to the principles shown in section 2 .

\subsection{Automatic tuning by PLL}

The performance of the PLL described in section 3 was tested experimentally. Figure 11 shows the process by which the frequency of the harmonic force approaches the target frequency. The initial frequency of $40[\mathrm{~Hz}]$ converged to the target frequency of $19.2[\mathrm{~Hz}]$ in 15 [s]. Therefore, the measuring conditions were automatically realized by the PLL.

\subsection{Mass measurement}

Mass measurement was performed while the PLL was operated. The primary system damping $c_{p}$ was reduced by the VCM. The damping $c_{a}$ was $66.37[\mathrm{Ns} / \mathrm{m}]$. The mass measurement results are shown in Table 2. The values are averages of 10 measurements. The average measurement error was within $0.21[\%]$.

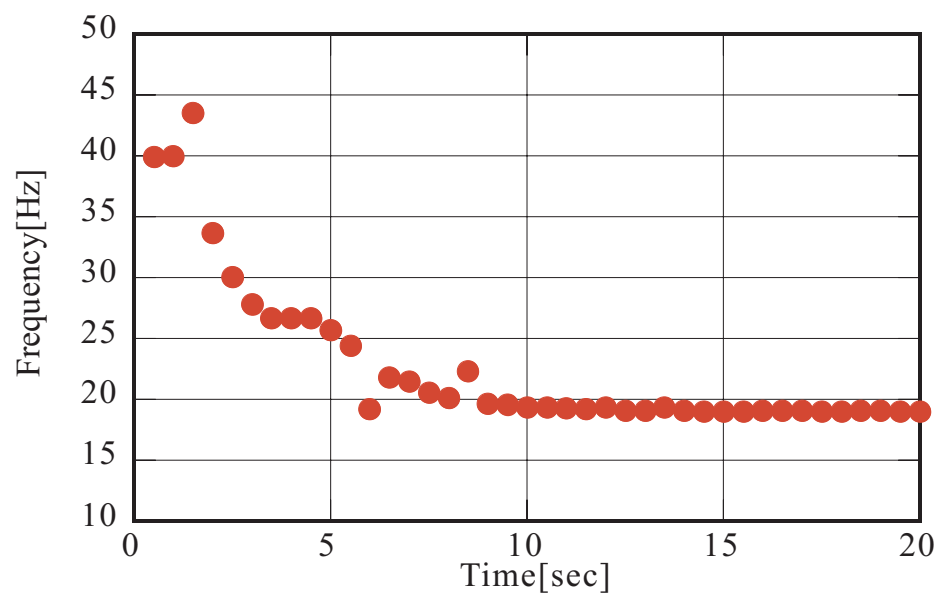

Fig.11 Automatic tuning by PLL

Table 2 Measurement results

\begin{tabular}{cccc}
\hline Actual mass $[\mathrm{g}]$ & Measured mass $[\mathrm{g}]$ & Calibrated value $[\mathrm{g}]$ & Error after calibration [\%] \\
\hline \hline 601.12 & 610.14 & 601.58 & 0.077 \\
606.20 & 615.96 & 607.32 & 0.185 \\
611.20 & 619.52 & 610.83 & -0.061 \\
616.39 & 626.44 & 617.65 & 0.204 \\
621.39 & 630.18 & 621.34 & -0.008 \\
626.46 & 635.10 & 626.19 & -0.043 \\
631.46 & 640.96 & 631.96 & 0.079 \\
635.74 & 644.80 & 635.75 & 0.002 \\
640.73 & 649.41 & 640.29 & -0.069 \\
645.82 & 655.37 & 646.17 & 0.054 \\
\hline
\end{tabular}




\section{Conclusions}

A new method of measuring mass was proposed; it is characterized by the use of a damped dynamic vibration absorber. A mass-spring system with a dynamic vibration absorber has a fixed point through which the frequency response curve passes regardless of damping in the absorber. The proposed method utilizes this fixed point to estimate mass independently of damping in the absorber. A measurement apparatus was designed and fabricated. The effect of damping in the absorber of the fabricated apparatus was considered. A difference in frequency between the fixed points of the gain and the phase was observed. The effect of the primary system damping was examined, and the measurement conditions were achieved by reducing damping in the primary system as much as possible. The measuring conditions were automatically realized by the PLL. Mass measurement was performed while the PLL was operated; the average measurement error was within 0.21 [\%].

\section{References}

[1] Oakey, W.E. and Lorenz, R. Survey and Experimental Testing of Non gravimetric Mass Measurement Devices, NASA-CR-151427, N77-26456 (1977).

[2] Mizuno, T., Device for Mass Measurement under Weightless Conditions Proceedings of the Society of Instrument and Control Engineers Annual Conference 2003 in Fukui, (2003), pp.245-251.

[3] Mizuno, T., Mass Measurement under Weightless Conditions with a Dynamic Vibration Absorber Proceedings of the 33rd Society of Instrument and Control Engineers Annual Conference, (1994), pp.729-734.

[4] Mizuno, T. and Araki, K., Mass Measurement Using a Dynamic Vibration Absorber under Weightless Conditions (in Japanese), Transactions of the Society of Instrument and Control Engineers, Vol.32, No.87 (1996), pp.1145-1151.

[5] Mizuno, T., Application of Dynamic Vibration Absorbers to Mass Measurement System, Proceedings of the 1997 American Society of Mechanical Engineers Design Engineering Technical Conferences, VIB-3803 (1997).

[6] Mizuno, T. and Negishi, T., Vibration-type Mass Measurement System with a Dynamic Vibration Absorber, Proceedings of XV IMEKO World Congress, Vol. III (1999), pp.33-38.

[7] Mizuno, T., Negishi, T. and Araki, K., New Principles of Mass Measurement Using Dynamic Vibration Absorbers, Proceedings of the 4th International Conference on Motion and Vibration Control, (1998), pp.237-342.

[8] Mizuno, T. and Araki, K., Application of Active Vibration Control Techniques to Mass Measurement, Proceedings of the 1998 IEEE International Conference on Control Applications, Vol. 1(1998), pp.609-613.

[9] Mizuno, T. and Sato, S., Vibration-type Mass Measurement System with an Undamped Dynamic Vibration Absorber (in Japanese), Transactions of the Japan Society of Mechanical Engineers, Series C, Vol.68,No.665, (2002), pp.37-43.

[10] Mizuno, T. and Sato, S., Mass Measurement System Using an Undamped Dynamic Vibration Absorber for Weightless Conditions, Proceedings of the American Society of Mechanical Engineers 2003 Design Engineering Technical Conferences and Computer and Information in Engineering Conference, DETC2003/VIB48552 (2003).

[11] Hartog J.P.D., Mechanical Vibration (1956), McGraw-Hill.

[12] Best R.E., Phase-Locked Loops: Design, Simulation and Applications (3rd ed.) (1997), McGraw-Hill.

[13] Moore, A.W.: Phase-Loocked loops for motor speed control, Institute of Electronical and Electronics Engineers Spectrum, (April 1973), pp.61-67.

[14] Mizuno, T. , Ishii, T. and Araki, K., Self-sensing Magnetic Suspension Using Hysteresis Amplifiers, Control Engineering Practice, Vol.6, No.9 (1998), pp.1133-1140. 